\title{
On the structure of the semigroup of entire étale mappings
}

\author{
Ronen Peretz
}

June 19, 2018

\section{Introduction}

This is a paper in analysis. In fact it deals with entire functions in a single complex variable which are local homeomorphisms of the complex plane. Also a central tool used is the so called composition operators. However, the motivation for the proposed research originates in the two-dimensional Jacobian Conjecture. The Jacobian Conjecture is one of the most famous and prominent conjectures in algebraic geometry [1, 5, 21]. It is mentioned in the list of open problems prepared by Steve Smale [21] that intends to orient mathematical research in this century (parallel to the famous list of problems prepared by David Hilbert at the beginning of the previous century). Thus, any significant progress in this problem is expected to make an important contribution to mathematical science. If it is true, then the Jacobian Conjecture gives a remarkably simple necessary and sufficient condition on a polynomial mapping $F: \mathbb{C}^{n} \rightarrow \mathbb{C}^{n}$ to be an automorphism [4, 5]. An automorphism is an invertible mapping $\mathbb{C}^{n} \rightarrow \mathbb{C}^{n}$ (i.e. an injective and a surjective mapping whose inverse $F^{-1}: \mathbb{C}^{n} \rightarrow \mathbb{C}^{n}$ is also polynomial). These polynomial mappings are precisely the morphisms that preserve the algebro-geometric affine structure of $\mathbb{C}^{n}$, which is a central theme in affine algebraic geometry. This is one instance of the importance of the conjecture.

Let us denote by $J(F)$ or by $J_{F}$ the Jacobian matrix of the mapping $F$. If $F$ is invertible, then in particular it is locally invertible and hence by the Inverse Mapping Theorem the determinant of its Jacobian matrix, det $J(F)$, does not vanish at any point of $\mathbb{C}^{n}$. However, the fact that $F$ is a polynomial mapping implies that $\operatorname{det} J(F)$ is a polynomial over the complex field $\mathbb{C}$ and hence the Fundamental Theorem of Algebra implies that det $J(F)$ must be a non-zero constant. This elementary argument proves that a necessary condition for a polynomial mapping $F$ to be an automorphism of $\mathbb{C}^{n}$, i.e. 
$F \in \operatorname{Aut}\left(\mathbb{C}^{n}\right)$, is that $\operatorname{det} J(F) \in \mathbb{C}^{\times}$. The Jacobian Conjecture speculates the validity of the inverse statement. Thus the Jacobian Conjecture is

$$
F \in \operatorname{Aut}\left(\mathbb{C}^{n}\right) \Leftrightarrow \operatorname{det} J(F) \in \mathbb{C}^{\times} .
$$

This is true for dimension $n=1$ but it is wide open for dimension $n \geq 2$. The original version of the conjecture was stated by Ott Keller in 1939 [10]. Keller worked over the integers $\mathbb{Z}$ and with polynomial mappings $F: \mathbb{Z}^{n} \rightarrow \mathbb{Z}^{n}$ that satisfy the unimodular Jacobian condition $\operatorname{det} J(F)=$ 1 or $(-1)$. Since that time, much research has been done on the so-called Keller Problem, giving rise to great many beautiful ideas and theories. Notable results are the degree reduction theorems [1, 2, 3, 22, These are based on $K$-theoretic principles that allow us, for example, to reduce the proof of the conjecture to the seemingly simple case of mappings of the form $F=\left(X_{1}, \ldots, X_{n}\right)+H\left(X_{1}, \ldots, X_{n}\right)$, where $\left(X_{1}, \ldots, X_{n}\right)$ is the identity mapping and $H\left(X_{1}, \ldots, X_{n}\right)$ is a cubic homogeneous mapping. However, one should prove the Jacobian Conjecture for such cubic mappings in an arbitrary dimension. We also note that the conjecture is known to be true in any dimension and for mappings of degree $\operatorname{deg} F \leq 2$, 1, 5]. So it seems that "we are almost there". Yet the degree 3 case seems to be out of our reach at least for the present.

Some experts in this field tend to believe that the general conjecture $(n \geq 2)$ might be false but that the two-dimensional case is possibly true. We mention here two pivotal two dimensional results. The first is a theorem of Moh [12, which asserts the validity of the conjecture for $n=2$ and degree $d=\operatorname{deg} F \leq 100$ or so. This is a difficult result. The second result is the ingenious counterexample of Pinchuk [17] to the so-called Real Jacobian Conjecture [18; namely, if $F: \mathbb{R}^{2} \rightarrow \mathbb{R}^{2}$ is a real polynomial mapping that satisfies the real Jacobian condition $\operatorname{det} J_{F}(X, Y) \neq 0 \forall(X, Y) \in \mathbb{R}^{2}$, then $F^{-1}$ exists. We note that in this case $\operatorname{det} J_{F}$ need not be a non-zero constant, but that the conclusion is also weaker, namely, $F^{-1}$ does not need to be a polynomial mapping. This natural real version of the original Jacobian Conjecture was open for sometime until in 1993 Pinchuk found a counterexample. His original clever construction gave rise to a degree 35 counterexample, but almost immediately it was reduced to a degree 25 counterexample. It is interesting to mention that the minimal degree of a counterexample is still not known, but we do have some lower bounds $(d \geq 7)$.

Another approach to solving the Jacobian Conjecture is via a thorough analysis of the structure of the semigroup of all the normalized étale mappings on $\mathbb{C}^{n}$.This approach is outlined in the papers of Kambyashi [7, 8, 9] 
and of the author 15, 16. The set of all the normalized étale mappings on $\mathbb{C}^{n}$ of degree $d$ or less can naturally be parametrized on a finite dimensional algebraic space, the so called Jacobian variety, $J(n, d)$, of degree $d$. Taking the union of these varieties gives us an object $J(n)$, which is known in the literature as an ind-variety. There is a canonical way to define a topology on $J(n)$. We call $J(n)$ the Jacobian variety ( of $\mathbb{C}^{n}$ ). As shown in the above papers, this structure might lead us to make a progress on the Jacobian Conjecture. Moreover, it is apparent (see [13]) that using this theory to solve the Jacobian Conjecture is intimately related to the singular loci $S(J(n, d))$ of the finite dimensional varieties. Equally relevant to the conjecture might be the singular locus $S(J(n))$ of the total ind-variety. However, it is still a challenge in the theory of ind-varieties to give a satisfactory definition of the smooth points. An early attempt by Shafarevich [19, 20] did not work well, as indicated by the clever example of Totatro [7, 8. Still it is possible that Shafarevich's definition will work well for our particular ind-variety $J(n)$. A good source to consult for singular ind-varieties is the book [11] of Kumar.

In our proposed research we hope to overcome that difficulty of attacking the Jacobian Conjecture via the structure of ind-varieties, at least in the two-dimensional case. We plan to do that by imposing a different structure on the family of the two-dimensional étale mappings. This structure is a geometric structure, as opposed to the algebraic ind-variety structure. Our proposed structure is a fractal structure where the role of the degree filter in the ind-variety structure, will be taken by self-similarity structure that is induced by the binary operation of the semigroup, i.e. the composition operators. We have two of these operators, a left composition operator and a right composition operator. They have different properties!

We hope to be able (in the future) to carefully analyze this structure and to tie the Jacobian Conjecture in dimension two to certain Zeta functions, thereby invoking a powerful arithmetic machinery to handle the two dimensional Jacobian Conjecture. Let us denote by et $\left(\mathbb{C}^{2}\right)$ the semigroup of two dimensional Keller mappings. We would like to prove something like the following:

a) That there exists an infinite index set $I$, and a family of mappings indexed by $I,\left\{F_{i} \mid i \in I\right\} \subset$ et $\left(\mathbb{C}^{2}\right)$ such that

$$
\operatorname{et}\left(\mathbb{C}^{2}\right)=\operatorname{Aut}\left(\mathbb{C}^{2}\right) \cup \bigcup_{i \in I} R_{F_{i}}\left(\operatorname{et}\left(\mathbb{C}^{2}\right)\right)
$$

where if $i \neq j$ then $R_{F_{i}}\left(\operatorname{et}\left(\mathbb{C}^{2}\right)\right) \cap R_{F_{j}}\left(\operatorname{et}\left(\mathbb{C}^{2}\right)\right)=\emptyset$.

b) That the parallel representation to the representation described in (a) 
above holds true, this time with respect to the left composition operators $L_{G_{j}}$.

These two claims will be the basis for a fractal structure on et $\left(\mathbb{C}^{2}\right)$ because the pieces $R_{F_{i}}\left(\right.$ et $\left.\left(\mathbb{C}^{2}\right)\right)$ are similar to each other in the sense that they are homeomorphic, and we further have the scaling property of selfsimilarity, namely $R_{F}\left(\right.$ et $\left.\left(\mathbb{C}^{2}\right)\right)$ is homeomorphic to its proper subspace

$R_{G \circ F}\left(\right.$ et $\left.\left(\mathbb{C}^{2}\right)\right)$ that is homeomorphic to its proper subspace $R_{H \circ G \circ F}\left(\right.$ et $\left.\left(\mathbb{C}^{2}\right)\right)$ etc. This is the right place to remark that the purpose of the current paper is to start and develop the parallel theory for entire functions in one complex variable. Results in this setting will hint that there are hopes to accomplish the above objective.

But our construction goes even further than this! The structure we propose to investigate is far more general than the structure of ind-varieties outlined in Kambyashi's papers [7, 8, 9]. The filtration in the ind-variety structure is an algebraic degree filtration. Thus it makes sense only in the algebraic context of polynomial mappings (as in the Jacobian Conjecture). However, the geometric structure that we suggest relies only on the fact that the underlined semigroup is a semigroup of local diffeomorphisms and is not necessarily algebraic. Thus, our theory applies to a much broader context of mappings. In addition, in that universe, we hope to be able to relate the semigroup structure that we suggest to Hausdorff measures and dimensions and to arithmetic. Thus we expect that our theory will also make a contribution in the opposite direction; namely, starting from a relatively well-understood semigroup of local diffeomorphisms, we will set out to conclude non-trivial theorems in the theories of Hausdorff measures, in fractals and in arithmetic.

The purpose of this paper is to deal with a semigroup very different from the semigroup of étale polynomial mappings on $\mathbb{C}^{2}$; namely, we will start to investigate the normalized semigroup of entire functions in one complex variable that has a nonvanishing first order derivative. This semigroup is better understood than the algebraic one. For example, we can (and will) invoke Nevanlinna's value distribution theory for entire functions in one complex variable. In the future we will try to understand what features are common to this analytic semigroup and to the algebraic semigroup that is suitable for the two-dimensional Jacobian Conjecture. A few surprises are to be expected. The reason is that our intuition will be built upon the analytic semigroup and so will lead us to expect similar properties in the algebraic setting. However, we might find out that not always will the algebraic semigroup have the same properties as the analytic semigroup. 


\section{The semigroup of the entire local homeomor- phisms in one complex variable, normalized at the origin}

\section{Definition 2.1.}

$$
\begin{gathered}
\operatorname{elh}(\mathbb{C})=\left\{f: \mathbb{C} \rightarrow \mathbb{C} \mid f \text { is entire, } \forall z \in \mathbb{C} f^{\prime}(z) \neq 0, f^{\prime}(0)=1\right\}, \\
\operatorname{Aut}(\mathbb{C})=\{z+a \mid a \in \mathbb{C}\} .
\end{gathered}
$$

Proposition 2.2. 1) $(\mathrm{elh}(\mathbb{C}), \circ)$ is a semigroup with an identity (o is composition).

2) If $\left\{f_{n}\right\}$ is a sequence in $\mathrm{elh}(\mathbb{C})$ that converges to $f$ uniformly on compact subsets of $\mathbb{C}$, then $f \in \operatorname{elh}(\mathbb{C})$.

3) If $\left\{f_{n}\right\}$ is a sequence in $\operatorname{elh}(\mathbb{C})$ that satisfies the Cauchy condition uniformly on compact subsets of $\mathbb{C}$, then $\lim f_{n} \in \operatorname{elh}(\mathbb{C})$.

4) $(\operatorname{Aut}(\mathbb{C}), \circ)$ is a closed group in $(\operatorname{elh}(\mathbb{C}), \circ)$ with respect to the topology of local uniform convergence (i.e. convergence uniformly on compact subsets of $\mathbb{C})$.

5) $f \in \operatorname{elh}(\mathbb{C})-\operatorname{Aut}(\mathbb{C}) \Leftrightarrow A(f) \neq \emptyset$ (See remark 2.3 below).

6) $\forall f \in \operatorname{elh}(\mathbb{C}), \forall z \in \mathbb{C}$ the fiber $f^{-1}(z)$ is a discrete subset of $\mathbb{C}$.

7) $\forall f \in \operatorname{elh}(\mathbb{C}),|\mathbb{C}-f(\mathbb{C})|<2$.

Remark 2.3. We recall that the asymptotic variety of $f, A(f)$, mentioned in part 5 of the proposition is defined as follows:

$$
\begin{gathered}
A(f)=\left\{\alpha \in \mathbb{C} \mid \exists \gamma:[0, \infty) \rightarrow \mathbb{C} \text { continuous such that } \lim _{t \rightarrow \infty}|\gamma(t)|=\infty\right. \\
\text { and } \left.\lim _{t \rightarrow \infty} f(\gamma(t))=\alpha\right\} .
\end{gathered}
$$

We don't use the sequential asymptotic variety $A_{S}(f)$ :

$$
A_{S}(f)=\left\{\alpha \in \mathbb{C} \mid \exists\left\{z_{n}\right\} \subseteq \mathbb{C} \text {, such that } \lim \left|z_{n}\right|=\infty \text { and } \lim f\left(z_{n}\right)=\alpha\right\} .
$$

The reason will soon be explained. We clearly have: $A(f) \subseteq A_{S}(f)$. Also $A(f)$ contains the set of all the $f$-Picard values, $\mathbb{C}-f(\mathbb{C})$.

\section{A proof of Proposition 2.2.}

1) By the chain rule, $f, g \in \operatorname{elh}(\mathbb{C}) \Rightarrow f \circ g \in \operatorname{elh}(\mathbb{C})$. The identity is $\operatorname{id}(z): \equiv z$.

2) By Cauchy's Theorem, $f=\lim f_{n}$ is an entire function. By Cauchy's estimate, $f^{\prime}=\lim f_{n}^{\prime}$ locally uniformly. By Hurwitz Theorem either $f^{\prime}(z) \equiv$ 
0 or $f^{\prime}$ never vanishes. By the normalization at the origin, $f^{\prime}(0)=1$ and hence $f \in \operatorname{elh}(\mathbb{C})$.

3) The assumption is that $\forall K \subset \mathbb{C}$ a compact, and $\forall \epsilon>0 \exists N_{\epsilon}(K)$ such that $\forall n, m \in \mathbb{Z}^{+}$, we have

$$
n, m>N_{\epsilon}(K) \Rightarrow \forall z \in K,\left|f_{n}(z)-f_{m}(z)\right|<\epsilon .
$$

So $\left\{f_{n}\right\}$ converges locally uniformly on $\mathbb{C}$ and the conclusion follows by part 2.

4) Clearly $(\operatorname{Aut}(\mathbb{C}), \circ)$ is a group. If $\left\{z+a_{n}\right\}$ converges locally uniformly then $a=\lim a_{n} \in \mathbb{C}$ exists and $\lim \left(z+a_{n}\right)=z+a \in \operatorname{Aut}(\mathbb{C})$.

5) By Hadamard's Theorem, [6], $f \in \operatorname{elh}(\mathbb{C})-\operatorname{Aut}(\mathbb{C}) \Leftrightarrow A(f) \neq \emptyset$.

6) If $z_{0} \in \mathbb{C}, f \in \operatorname{elh}(\mathbb{C})$ and $f^{-1}\left(z_{0}\right)$ is not a discrete subset of $\mathbb{C}$, then $\exists\left\{w_{n}\right\} \subseteq f^{-1}\left(z_{0}\right)$ of different points, such that $w_{0}=\lim w_{n}$ exists. Hence $\forall n \in \mathbb{Z}^{+}, f\left(w_{n}\right)=z_{0}$ and by the permanence principle $f(z) \equiv z_{0}$ which contradicts $f \in \operatorname{elh}(\mathbb{C})$.

7) This is the Picard's Little Theorem.

We now explain why we do not use $A_{S}(f)$.

Proposition 2.4. If $f(z)$ is an entire non-polynomial function, then $A_{S}(f)=$ $\mathbb{C}$.

\section{Proof.}

Let $z_{0} \in \mathbb{C}$ and let $\left\{w_{n}\right\} \subseteq f(\mathbb{C})$ satisfy $\lim w_{n}=z_{0}$. Since $f$ is nonpolynomial it has infinitely many regular maximal domains $\Omega_{n}$ that tile up the complex plane, [13. We choose $\forall n \in \mathbb{Z}^{+}, z_{n} \in \Omega_{n}$ such that $w_{n}=f\left(z_{n}\right)$. By passing to a subsequence, if necessary, we may assume that $\lim \left|z_{n}\right|=\infty$. Hence $z_{0} \in A_{S}(f)$.

\section{The right and the left mappings on $\operatorname{elh}(\mathbb{C})$}

Definition 3.1. Let $f \in \operatorname{elh}(\mathbb{C})$. The right mapping on elh $(\mathbb{C})$, induced by $f$ is defined by:

$$
\begin{aligned}
& R_{f}: \operatorname{elh}(\mathbb{C}) \rightarrow \operatorname{elh}(\mathbb{C}) \\
& R_{f}(g)=g \circ f .
\end{aligned}
$$

The left mapping on elh $(\mathbb{C})$, induced by $f$ is defined by:

$$
\begin{aligned}
& L_{f}: \operatorname{elh}(\mathbb{C}) \rightarrow \operatorname{elh}(\mathbb{C}) \\
& L_{f}(g)=f \circ g .
\end{aligned}
$$


Definition 3.2. Let $g \in \operatorname{elh}(\mathbb{C})$. We will denote by $T_{f}(g)$ the set of all the finite asymptotic tracts of $g(z)$. Thus formally:

$T_{f}(g)=\left\{\gamma:[0, \infty) \rightarrow \mathbb{C} \mid \gamma\right.$ continuous, $\lim _{t \rightarrow \infty}|\gamma(t)|=\infty$ and $\left.\lim _{t \rightarrow \infty} g(\gamma(t)) \in \mathbb{C}\right\}$.

We did not include in $T_{f}(g)$ the asymptotic tracts of $g$ of the infinite asymptotic value, i.e. those tracts $\gamma:[0, \infty) \rightarrow \mathbb{C}$ which are continuous and satisfy $\lim _{t \rightarrow \infty}|\gamma(t)|=\infty$ and $\lim _{t \rightarrow \infty}|g(\gamma(t))|=\infty$. We collect together asymptotic tracts of $g$ according to the following criterion.

Definition 3.3. Two finite asymptotic tracts $\gamma, \beta \in T_{f}(g)$ of $g \in \operatorname{elh}(\mathbb{C})$ are said to be equivalent if:

a) $\lim _{t \rightarrow \infty} g(\gamma(t))=\lim _{t \rightarrow \infty} g(\beta(t))$.

b) The finite tracts $\gamma$ and $\beta$ are homotopic via a homotopy that fixes $\infty$. Thus $\exists H(s, t):[0,1] \times[0, \infty) \rightarrow \mathbb{C}$ continuous in $(s, t)$ and satisfying $H(0, t)=\gamma(t), H(1, t)=\beta(t) \forall t \geq 0$ and

$$
\left\{\begin{array}{l}
\lim _{t \rightarrow \infty}|H(s, t)|=\infty \forall 0 \leq s \leq 1 \\
\lim _{t \rightarrow \infty} f(H(s, t))=\lim _{t \rightarrow \infty} g(\gamma(t)) .
\end{array}\right.
$$

It is standard to conclude that the above relation on $T_{f}(g)$ is an equivalence relation. We denote by $T_{0 f}(g)$ the set of all the equivalence classes.

It is natural to ask if there is a canonical way to choose representatives of the classes in $T_{0 f}(g)$. We would be happy to obtain such a representative by a construction similar to the resolution process that was used in $\mathbb{C}[X, Y]^{2}$ (See [14). However, in trying to get the parallel of the asymptotic identities we find that the situation in $\operatorname{elh}(\mathbb{C})$ is harder. To explain we now prove the following proposition.

Proposition 3.4. Let $f(z)$ be a non-constant entire function.

1) If $g(z) \in H\left(0<\left|z-z_{0}\right| \leq r\right)-H\left(\left|z-z_{0}\right| \leq r\right)$, then $f(g(z)) \in H(0<$ $\left.\left|z-z_{0}\right| \leq r\right)-H\left(\left|z-z_{0}\right| \leq r\right)$.

2) If $h(z, w)$ has a non-removable singularity at $\left(z_{0}, w_{0}\right)$ then $f(h(z, w))$ can not be holomorphic in a neighborhood of $\left(z_{0}, w_{0}\right)$.

The symbol $H(\Omega)$ for an open non-empty $\Omega \subseteq \mathbb{C}$ stands for the family of holomorphic functions defined on $\Omega$.

Proof.

1) By the assumption $z_{0}$ is an isolated non-removable singularity of $g(z)$. Hence either $z_{0}$ is an essential singularity of $g(z)$ or it is a pole of $g(z)$. 
Case 1: $z_{0}$ is an essential singularity of $g(z)$. Then by the Picard Theorem, $g\left(\left\{0<\left|z-z_{0}\right| \leq r\right\}\right)=\mathbb{C}$ or it is $\mathbb{C}-\{a\}$ for a fixed $a \in \mathbb{C}$. If $f(g(z)) \in H\left(\left|z-z_{0}\right| \leq r\right)$ then $f\left(g\left(\left\{0<\left|z-z_{0}\right| \leq r\right\}\right)\right.$ had a compact closure and hence $f(\mathbb{C})$ or $f(\mathbb{C}-\{a\})$ had a compact closure. By the Liouville Theorem $f(z)$ had to be a constant, which contradicts our assumption on $f(z)$.

Case 2: $z_{0}$ is a pole of $g(z)$. In that case $\lim _{z \rightarrow z_{0}} g(z)=\infty$ and $g(\{0<$ $\left.\left.\left|z-z_{0}\right| \leq r\right\}\right)$ contains the complement of some disc, say $\{R<|w|\}$. If $f(g(z)) \in H\left(\left|z-z_{0}\right| \leq r\right)$ then $f(\{R<|w|\})$ had a compact closure and so also had $f(\mathbb{C})$. By Liuoville Theorem $f(z)$ had to be a constant, which is a contradiction.

2) In this case we can either fix $w=w_{0}^{\prime}$ and consider $f\left(h\left(z, w_{0}^{\prime}\right)\right)$ or fix $z=z_{0}^{\prime}$ and consider $f\left(h\left(z_{0}^{\prime}, w\right)\right)$ and then use part 1 .

So we can not expect naive asymptotic identities in the holomorphic one variable case, which will resemble the two variable polynomial case. For general entire functions the asymptotic variety $A(f)$ can be pretty wild. For instance it could be any finite subset of $\mathbb{C}$, and it could be as large as the whole space, $\mathbb{C}$.

Proposition 3.5. If $f, g \in \operatorname{elh}(\mathbb{C})$ then $T_{f}(g) \subseteq T_{f}(f \circ g), f(A(g)) \subseteq A(f \circ g)$

\section{Proof.}

$\gamma \in T_{f}(g) \Rightarrow \gamma:[0, \infty) \rightarrow \mathbb{C}$ is continuous, $\lim _{t \rightarrow \infty}|\gamma(t)|=\infty$ and $\lim _{t \rightarrow \infty} g(\gamma(t)) \in \mathbb{C} \Rightarrow \lim _{t \rightarrow \infty} f(g(\gamma(t))) \in \mathbb{C} \Rightarrow \lim _{t \rightarrow \infty}(f \circ g)(\gamma(t)) \in$ $\mathbb{C} \Rightarrow \gamma \in T_{f}(f \circ g)$.

$a \in f(A(g)) \Rightarrow \exists \gamma \in T_{f}(g)$ such that $a=\lim _{t \rightarrow \infty} f(g(\gamma(t))) \Rightarrow \exists \gamma \in$ $T_{f}(f \circ g)$ such that $a=\lim _{t \rightarrow \infty}(f \circ g)(\gamma(t)) \Rightarrow a \in A(f \circ g)$.

This proposition tells us that left composition of functions in elh $(\mathbb{C})$ does nor decrease $T_{f}(g)$ ( $g$ the right function), the set of all the finite asymptotic tracts of the right factor and consequently it does not decrease the left image of its finite asymptotic values. We ask, when in Proposition 3.5 we have a strict containment? This happens exactly when $\left.\exists \gamma \in T_{f}(f \circ g)-T_{(} g\right)$. This implies that $a=\lim _{t \rightarrow \infty}(f \circ g)(\gamma(t)) \in \mathbb{C}$ but $\lim _{t \rightarrow \infty} g(\gamma(t))$ does not exist. If by "does not exist" we insist that $\lim _{t \rightarrow \infty} g(\gamma(t))$ does not exist as a finite number, then of course we can take $g(z)=z$ and $\gamma \in T_{f}(f)$. However, if we make no distinction between finite limits and an infinite limit then this example no longer works. Thus the following is worth mentioning.

Proposition 3.6. If $f, g \in \operatorname{elh}(\mathbb{C})$, then $\gamma \in T_{f}(f \circ g)$ implies that either $\gamma \in T_{f}(g)$ or else $\lim _{t \rightarrow \infty} g(\gamma(t))=\infty$. 


\section{Proof.}

Of course, it is enough to take $\gamma \in T_{f}(f \circ g)-T_{f}(g)$ and prove that $\lim _{t \rightarrow \infty} g(\gamma(t))=\infty$. If this is not the case then $\lim _{t \rightarrow \infty} g(\gamma(t))$ does not exist in the broad sense (i.e. not as a finite number nor $\infty$ ). Hence we have two sequences $t_{n}, s_{n} \in[0, \infty)$ so that $\lim t_{n}=\lim s_{n}=\infty$ and $a=\lim g\left(\gamma\left(t_{n}\right)\right)$, $b=\lim g\left(\gamma\left(s_{n}\right)\right)$ exist and are not equal to one another. But by our assumption $\gamma \in T_{f}(f \circ g)$ and so $f(a)=f(b)$. We claim that there exists a sequence $\left\{a_{n}\right\} \subseteq \mathbb{C}$ of distinct points for which $\lim a_{n}=a$ and $\forall n, f\left(a_{n}\right)=f(a)$. If indeed this is true then by the permanence principle $f(z) \equiv f(a)$ a constant. This contradicts the assumption that $f \in \operatorname{elh}(\mathbb{C})$. To prove the existence of the sequence $\left\{a_{n}\right\}$ we prove the following: $\forall 0<r<|b-a|$ $\exists z_{r} \in\{z \in \mathbb{C}|| z-a \mid=r\}$ so that $f\left(z_{r}\right)=f(a)$. This will suffice, for we can take $a_{n}=z_{r_{n}}$ where $r_{n}=|b-a| / 2^{n}$. So we fix $r, 0<r<|b-a|$. We recall that $\lim _{t \rightarrow \infty} f(g(\gamma(t)))=f(a)=f(b), \lim g\left(\gamma\left(t_{n}\right)\right)=a, \lim g\left(\gamma\left(s_{n}\right)\right)=b$, $\lim t_{n}=\lim s_{n}=\infty$. So the curve $g(\gamma(t)), t \in[0, \infty)$ goes back and forth between points that are as close as we please to $a$ or to $b$, and for large $t$, $f$ maps this curve to a neighborhood of $f(a)$ and this neighborhood can be as small as we please. Taking the intersection points of the curve $g(\gamma(t))$ with the circle $|z-a|=r$ (we recall that $0<r<|b-a|$ ) we see that $\exists$ a sequence $\alpha_{n}$ on this circle such that $\alpha=\lim \alpha_{n}$ exists and $f(\alpha)=f(a)$. We take $z_{r}=\alpha$.

Remark 3.7. We note that our proof of Proposition 3.6 uses the fact that $f \in \operatorname{elh}(\mathbb{C})$, but $g$ can be less restricted. In fact $g$ can be only a continuous function.

An important consequence comes out of Proposition 3.6 and out of its proof. This will be a result that sharpens Proposition 3.5. Namely,

Theorem 3.8. If $f, g \in \operatorname{elh}(\mathbb{C})$, then $A(f \circ g)=A(f) \cup f(A(g))$.

\section{Proof.}

By Proposition 3.5 we have $f(A(g)) \subseteq A(f \circ g)$. If $a \in A(f \circ g)-f(A(g))$ then there exists an asymptotic tract $\gamma \in T_{f}(f \circ g)-T_{f}(g)$ so that $\lim _{t \rightarrow \infty} f(g(\gamma(t)))=$ a. By Proposition $3.6 \lim _{t \rightarrow \infty} g(\gamma(t))=\infty$. Hence $g(\gamma(t)) \in T_{f}(f)$ and $a \in A(f)$. This proves that, $A(f \circ g)-f(A(g)) \subseteq A(f)$. Finally, if $a \in A(f)-A(f \circ g)$ then there is an asymptotic tract $\delta \in T_{f}(f)$, i.e. $\lim _{t \rightarrow \infty} \delta(t)=\infty$ and $\lim _{t \rightarrow \infty} f(\delta(t))=a$. But (since $a \notin A(f \circ g)$ ) there is no $\gamma(t) \in T_{f}(f \circ g)$ for which $\lim _{t \rightarrow \infty} f(g(\gamma(t)))=a$. To get a contradiction (and thus to prove that there is no $a \in A(f)-A(f \circ g)$ ) we consider the pre-image $g^{-1}(\delta)$. Each component $\beta$ of this curve can not be bounded because $\delta \triangle g(\beta)$ is a finite set and $\delta$ is unbounded. If $\lim _{t \rightarrow \infty} \beta(t) \neq \infty$ 
then as in the proof of Proposition 3.6 we deduce that $f \circ g \equiv$ Const.. Hence not both $f, g \in \operatorname{elh}(\mathbb{C})$. We conclude that $\lim _{t \rightarrow \infty} \beta(t)=\infty$ and $\lim _{t \rightarrow \infty} f(g(\beta(t)))=a$. But this means that $a \in A(f \circ g)$ which contradicts our assumption that $a \in A(f)-A(f \circ g)$.

Remark 3.9. We see that if $f, g \in \operatorname{elh}(\mathbb{C})$ and $a \in A(f)$ and $\delta \in T_{f}(f)$ is an $a$-asymptotic tract, then for each irreducible component $\beta$ of the curve $g^{-1}(\delta)$ either $\beta \in T_{f}(g)$ or $g(\beta(t)) \in T_{f}(f)$ is an $a$-asymptotic tract of $f$.

Proposition 3.10. Let $f \in \operatorname{elh}(\mathbb{C})$. If $\exists g \in \operatorname{elh}(\mathbb{C})$ such that $T_{f}(g)=$ $T_{f}(f \circ g)$, then $f(\mathbb{C})=\mathbb{C}$.

\section{Proof.}

Since $f \in \operatorname{elh}(\mathbb{C})$ we have $\mathbb{C}-f(\mathbb{C}) \subseteq A(f)$ because the only points in the complement of the image of $f$ are the Picard exceptional values of $f$ which are also asymptotic values of $f$. Of course $f$ may have at most one such a value. If $T_{f}(g)=T_{f}(f \circ g)$ then by Theorem 3.8 we must have $A(f) \subseteq f(A(g)) \subseteq f(\mathbb{C})$. Thus there are no Picard exceptional values of $f$.

The last proposition implies that if $f \in \operatorname{elh}(\mathbb{C})$ is not a surjective mapping then $\forall g \in \operatorname{elh}(\mathbb{C})$ we must have $T_{f}(g) \subset T_{f}(f \circ g)$. In particular $T_{f}(f) \subset$ $T_{f}(f \circ f)$. But also $f \circ f$ is not surjective because $(f \circ f)(\mathbb{C}) \subseteq f(\mathbb{C}) \subset \mathbb{C}$. Hence $T_{f}(f \circ f) \subset T_{f}(f \circ f \circ f)$. By induction on the number of compositions we get the infinite strictly ascending sequence,

$$
T_{f}(f) \subset T_{f}(f \circ f) \subset T_{f}(f \circ f \circ f) \subset \ldots \subset T_{f}(f \circ f \circ \ldots \circ f) \subset \ldots
$$

Another consequence of Theorem 3.8, is that for any $f \in \operatorname{elh}(\mathbb{C})$ we have the identity,

$$
A\left(f^{\circ(n+1)}\right)=A(f) \cup \bigcup_{k=1}^{n} f^{\circ k}(A(f)) .
$$

To see that we use induction as follows $A(f) \cup f(A(f))=A(f \circ f)$, hence $A(f) \cup f(A(f)) \cup(f \circ f)(A(f))=A(f) \cup f(A(f) \cup f(A(f)))=A(f) \cup f(A(f \circ$ $f))=A(f \circ f \circ f)$, and so on.

Proposition 3.11. The following are equivalent:

1) $f \notin \operatorname{Aut}(\mathbb{C})$.

2) $R_{f}(\operatorname{elh}(\mathbb{C})) \subset \operatorname{elh}(\mathbb{C})-\operatorname{Aut}(\mathbb{C})$.

3) $L_{f}(\operatorname{elh}(\mathbb{C})) \subset \operatorname{elh}(\mathbb{C})-\operatorname{Aut}(\mathbb{C})$. 
Proof.

This follows by Theorem 3.8 and Proposition 3.5 which imply that:

$$
T_{f}\left(R_{f}(g)\right)=T_{f}(g \circ f) \supseteq T_{f}(f), A\left(L_{f}(g)\right)=A(f \circ g) \supseteq A(f),
$$

and by Proposition 2.2 part 5 , which implies that $f \notin \operatorname{Aut}(\mathbb{C})$ is equivalent to $A(f) \neq \emptyset$ and so also to $T_{f}(f) \neq \emptyset$.

Proposition 3.12. $\forall f \in \operatorname{elh}(\mathbb{C}), R_{f}$ is injective.

\section{Proof.}

$R_{f}(g)=R_{f}(h) \Rightarrow g \circ f=\left.h \circ f \Rightarrow g\right|_{f(\mathbb{C})}=\left.h\right|_{f(\mathbb{C})} \Rightarrow g \equiv h$. The last implication follows by $|\mathbb{C}-f(\mathbb{C})| \leq 1$.

Proposition 3.13. $\forall f, g, h \in \operatorname{elh}(\mathbb{C}),\left(L_{f}(g)=L_{f}(h) \wedge g \not \equiv h\right) \Rightarrow \exists t(z)$ an entire function, such that $g(z)=h(z)+e^{t(z)}$.

Proof.

$L_{f}(g)=L_{f}(h) \Rightarrow f \circ g=f \circ h$. By $g \not \equiv h$ there are points $z \in \mathbb{C}$ for which $g(z) \neq h(z)$. Let $N=\{z \in \mathbb{C} \mid g(z) \neq h(z)\}$. We will prove that $N=\mathbb{C}$ and so $g(z)-h(z)$ never vanishes, so $\exists$ an entire function $t(z)$ such that $g(z)-h(z)=e^{t(z)}$. Thus $N \neq \emptyset$ is open in the strong topology. For $g, h$ are local homeomorphisms and if $g(z) \neq h(z)$ then $\exists O$, an open neighborhood of $z$ in the strong topology, such that $g(O) \cap h(O)=\emptyset$. Let $z \in \partial N$. Let $z_{n} \in N$ satisfy $\lim z_{n}=z$. Then $\forall n \in \mathbb{Z}^{+}, g\left(z_{n}\right) \neq h\left(z_{n}\right), f\left(g\left(z_{n}\right)\right)=f\left(h\left(z_{n}\right)\right)$ and $g(z)=h(z)$. This implies that in any strong neighborhood of $g(z)=h(z)$ there are distinct points $g\left(z_{n}\right) \neq h\left(z_{n}\right)$ (for $n \in \mathbb{Z}^{+}$large enough), so that $f\left(g\left(z_{n}\right)\right)=f\left(h\left(z_{n}\right)\right)$. Hence $f$ can not be injective in any strong neighborhood of the point $g(z)=h(z)$. This contradicts the assumption $f \in \operatorname{elh}(\mathbb{C})$ and this proves that $\partial N=\emptyset$ or equivalently that $N=\mathbb{C}$.

Surprisingly, elh $(\mathbb{C})$ behaves very different from ét $\left(\mathbb{C}^{2}\right)$, the two dimensional Keller mappings with respect to $L_{f}$. Namely in this case we have:

Proposition 3.14. $\exists f \in \operatorname{elh}(\mathbb{C})$, such $L_{f}$ is not injective.

\section{Proof.}

We take $f(z)=e^{2 \pi i z}, g(z)=e^{z}+1, h(z)=e^{z}$. Then $g \not \equiv h$ but $L_{f}(g)=$ $e^{2 \pi i\left(e^{z}+1\right)}=e^{2 \pi i e^{z}}=L_{f}(h)$.

Remark 3.15. Unless otherwise said our topology on elh $(\mathbb{C})$ will be that of local uniform convergence. Thus $f_{n} \rightarrow f$ in that topology if the sequence converges to $f$ uniformly on each compact subset $K \subset \mathbb{C}$. We recall (by Proposition 2.2 part 2) that in this case $f \in \operatorname{elh}(\mathbb{C})$. 
We recall the connection with the so-called compact-open topology. If $X$ and $Y$ are topological spaces, $C(X, Y)$ denotes the set of all the continuous mappings from $X$ to $Y$. If $K$ is a compact subset of $X$ and $U$ an open subset of $Y$, we define $S(K, U)=\{f: X \rightarrow Y \in C(X, Y) \mid f(K) \subset U\}$. The sets $S\left(K_{1}, \ldots, K_{n} ; U_{1}, \ldots, U_{n}\right)=\bigcap_{i=1}^{n} S\left(K_{i}, U_{i}\right), n \in \mathbb{Z}^{+}$, form a basis for a topology on $C(X, Y)$ called the compact-open topology. It is denoted by $\tau_{C O}$. The following facts are well known:

$\left.1_{C O}\right)$ In the compact-open topology, $C(X, Y)$ is a Hausdorff or regular space whenever $Y$ is a Hausdorff or regular space.

$2_{C O}$ ) If $X$ is locally compact and $X$ and $Y$ are second countable, then so is $\left(C(X, Y), \tau_{C O}\right)$.

$\left.3_{C O}\right)$ Let $X$ be locally compact and second countable, and let $Y$ be regular and second countable. Then $\left(C(X, Y), \tau_{C O}\right)$ is metrizable.

Let $(Y, d)$ be a metric space and let $X$ be a topological space. For a compact subset $K \subset X$ of $X, \varepsilon>0$, and $f \in Y^{X}$, we define $B_{K}(f, \varepsilon)=\{g$ : $X \rightarrow Y \mid d(f(x), g(x))<\varepsilon, \forall x \in K\}$. The sets $B_{K}(f, \varepsilon)$ form a basis for a topology on $Y^{X}$, called the topology of compact convergence. It is denoted by $\tau_{C C}$. Clearly $f_{n}$ converges to $f$ in $\tau_{C C}$ if and only if $\forall$ compact $K,\left.f_{n}\right|_{K}$ converges uniformly to $\left.f\right|_{K}$.

$\left.4_{C C-C O}\right) C(X, Y)$ is closed in $\left(Y^{X}, \tau_{C C}\right)$.

$\left.5_{C C-C O}\right)$ Let $X$ be a topological space and $Y$ a metric space. Then $\left(C(X, Y), \tau_{C O}\right)=$ $\left(C(X, Y), \tau_{C C}\right)$.

In particular, by $5_{C C-C O}$ we have $\left(\operatorname{elh}(\mathbb{C}), \tau_{C O}\right)=\left(\operatorname{elh}(\mathbb{C}), \tau_{C C}\right)$. Our natural topology on elh $(\mathbb{C})$ is $\tau_{C C}$, as mentioned before. Thus in this case it coincides with the compact-open topology, $\tau_{C O}$.

Proposition 3.16. 1) The topological space $\left(\operatorname{elh}(\mathbb{C}), \tau_{C C}\right)$ is path connected. 2) The image of the $f$-right mapping, $R_{f}(\mathrm{elh}(\mathbb{C}))$ is a closed subset of $\left(\operatorname{elh}(\mathbb{C}), \tau_{C C}\right)$. Here $f \in \operatorname{elh}(\mathbb{C})$ is fixed.

\section{Proof.}

1) Let us assume first that $f \in \operatorname{elh}(\mathbb{C})$ and also $f(0)=0$. Then $\forall 0<t \leq 1$, $f_{t}(z)=(1 / t) f(t z) \in \operatorname{elh}(\mathbb{C})$. Let us denoted $f_{0}(z)=\mathrm{id}(z)$. Then $f_{1}=f$ and $f_{0}=\mathrm{id}$ and $\left\{f_{t} \mid 0 \leq t \leq 1\right\}$ is a path in $\operatorname{elh}(\mathbb{C})$ from $f_{0}$ to $f$. In the general case we take $(1 / t)(f(t z)-f(0))+f(0)$ that connects $z+f(0)$ to $f(z)$. Now $\operatorname{Aut}(\mathbb{C})$ is path connected and we are done.

2) By $3_{C O}$ the space $\left(\operatorname{elh}(\mathbb{C}), \tau_{C O}\right)$ is metrizable. By $5_{C C-C O}$ it coincides with $\left(\operatorname{elh}(\mathbb{C}), \tau_{C C}\right)$ and so to prove the closedness of $R_{f}(\operatorname{elh}(\mathbb{C}))$ we can 
show that the limit point of any convergent sequence in $R_{f}(\mathrm{elh}(\mathbb{C}))$ belongs to $R_{f}(\operatorname{elh}(\mathbb{C}))$. That will be sufficient. Thus let $f_{n} \in R_{f}(\operatorname{elh}(\mathbb{C}))$ satisfy $f_{n} \rightarrow g$ in $\tau_{C C}$. By the definition of $R_{f}(\operatorname{elh}(\mathbb{C})) \forall n \in \mathbb{Z}^{+}, \exists g_{n} \in \operatorname{elh}(\mathbb{C})$ such that $f_{n}=g_{n} \circ f$. we have $g_{n} \circ f \rightarrow g$ in $\tau_{C C}$. So $\forall K \subset \mathbb{C}$ a compact and $\forall \varepsilon>0, \exists N=N(\varepsilon, K)$ such that $n, m>N \Rightarrow\left|g_{n}(f(z))-g_{m}(f(z))\right|<$ $\varepsilon \forall z \in K$. So $n, m>N \Rightarrow\left|g_{n}(w)-g_{m}(w)\right|<\varepsilon, \forall w \in K_{1}=f(K)$. This implies by Proposition 2.2 part 3 that $h=\lim g_{n} \in \operatorname{elh}(\mathbb{C})$ and hence $f_{n}=g_{n} \circ f \rightarrow h \circ f \in R_{f}(\operatorname{elh}(\mathbb{C}))$.

Remark 3.17. The mapping $R_{f}: \operatorname{elh}(\mathbb{C}) \rightarrow \operatorname{elh}(\mathbb{C})$ is continuous and an injective mapping (Proposition 3.12). The image $R_{f}(\mathrm{elh}(\mathbb{C})$ ) is a closed subset of $\left(\operatorname{elh}(\mathbb{C}), \tau_{C C}\right)$ (Proposition 3.16 part 2). Hence $R_{f}(\operatorname{elh}(\mathbb{C})$ ) is also open $\Leftrightarrow R_{f}(\operatorname{elh}(\mathbb{C}))=\operatorname{elh}(\mathbb{C})$ because by Proposition 3.16 part 1 the space $\left(\operatorname{elh}(\mathbb{C}), \tau_{C C}\right)$ is connected. This is equivalent to $f \in \operatorname{Aut}(\mathbb{C})$ by Proposition 3.11 .

Thus it is natural to ask the following: Let $f \in \operatorname{elh}(\mathbb{C})-\operatorname{Aut}(\mathbb{C})$. What is the boundary of the image $\partial R_{f}(\operatorname{elh}(\mathbb{C}))$ ? We note that since $R_{f}(\operatorname{elh}(\mathbb{C}))$ is closed in $\left(\operatorname{elh}(\mathbb{C}), \tau_{C C}\right)$, it follows that $\partial R_{f}(\operatorname{elh}(\mathbb{C})) \subseteq R_{f}(\operatorname{elh}(\mathbb{C}))$. Moreover, every automorphism $z+a$ is an interior point of the open set $\operatorname{elh}(\mathbb{C})-R_{f}(\operatorname{elh}(\mathbb{C}))$ because $z+a \notin R_{f}(\operatorname{elh}(\mathbb{C}))$. The reason is that if $z+a \in R_{f}(\operatorname{elh}(\mathbb{C}))$ then $g(f(z))=z+a$ for some $g \in \operatorname{elh}(\mathbb{C})$. This implies by Proposition 3.5 that $T_{f}(f) \subseteq T_{f}(z+a)$ which is an absurd since $T_{f}(f) \neq \emptyset$ while $T_{f}(z+a)=\emptyset$. So a boundary point $h \in \partial R_{f}(\operatorname{elh}(\mathbb{C}))$ has the form $h=g \circ f$ for some $g \in \operatorname{elh}(\mathbb{C})$ but there should exist a sequence $h_{n} \in \operatorname{elh}(\mathbb{C})-R_{f}(\operatorname{elh}(\mathbb{C}))$ such that $h_{n} \rightarrow h$ in $\tau_{C C}$. At most finitely many of the $h_{n} \in \operatorname{Aut}(\mathbb{C})$, because by the Theorem of Hurwitz Aut $(\mathbb{C})$ is closed in $\left(\operatorname{elh}(\mathbb{C}), \tau_{C C}\right)$. To try and obtain such a sequence $h_{n}$, we might want to see if we can pick its elements from among the family $h_{t}(z)=(1 / t) h(t z), 0<t \leq 1$. We assume here that $g(0)=f(0)=0$ for simplicity. We observe that $h_{t}(z)=(1 / t) g(f(t z))$. So the question boils down to: can it possibly be that $\forall 0<t<1$ we will have $h_{t}(z) \notin R_{f}(\operatorname{elh}(\mathbb{C}))$ ? More concretely, can we find a family $G(t, f(z)), 0<t<1$ of entire functions in $f(z)$ such that

$$
\frac{1}{t} g(f(t z))=G(t, f(z)), 0<t \leq 1 .
$$

We observe that the left hand side is entire both in $t$ and in $z$, and in fact there is a full symmetry between $t$ and $z$ in $g(f(t z))$ which indicates a symmetry in the power series coefficients (only the diagonal terms can be different from zero). In other words $t G(t, f(z))=\sum_{n=1}^{\infty} A_{n n} t^{n} z^{n}$. In fact $\forall n \in \mathbb{Z}^{+}, A_{n n}=\left.(1 / n !)\left(d^{n} / d z^{n}\right)\{g(f(z))\}\right|_{z=0}$. We can derive a first order 
linear pde on $G(u, v)$ that avoids the entire function $g(z)$, as follows:

$$
g(f(t z))=t G(t, f(z))
$$

applying $\partial_{z}$ we get,

$$
t f^{\prime}(t z) g^{\prime}(f(t z))=t f^{\prime}(z) G_{v}(t, f(z))
$$

hence,

$$
f^{\prime}(t z) g^{\prime}(f(t z))=f^{\prime}(z) G_{v}(t, f(z)) .
$$

On the other hand if we apply $\partial_{t}$ instead we get,

$$
z f^{\prime}(t z) g^{\prime}(f(t z))=G(t, f(z))+t G_{u}(t, f(z)) .
$$

Taking the obvious linear combination of the two last equations we arrive at our pde,

$$
G(t, f(z))+t G_{u}(t, f(z))-z f^{\prime}(z) G_{V}(t, f(z))=0 .
$$

As promised the last equation does not contain $g$. We have noted before that $G(t, f(z))$ is entire in $(t z)$ and so we deduce that $G(u, v)$ is entire in $u, v$. Let us assume that $G(u, v)=\sum_{n, m} B_{n m} u^{n} v^{m}$. We substitute this into into our pde and obtain,

$$
\begin{gathered}
0 \equiv G(t, f)+t G_{u}(t, f)-z f^{\prime} G_{v}(t, f)= \\
=\sum B_{n m} t^{n} f^{m}+\sum n B_{n m} t^{n} f^{m}-\frac{z f^{\prime}}{f} \sum m B_{n m} t^{n} f^{m}= \\
=\sum\left(\left(1+n-\frac{z f^{\prime}}{f} m\right) B_{n m} f^{m}\right) t^{n} .
\end{gathered}
$$

We deduce that $\forall n \in \mathbb{Z}^{+} \cup\{0\}$ we have the identities,

$$
\sum_{m}\left(1+n-\frac{z f^{\prime}}{f} m\right) B_{n m} f^{m} \equiv 0
$$

Let us fix an $n \in \mathbb{Z}^{+} \cup\{0\}$. Then

$$
(1+n) \sum_{m} B_{n m} f^{m} \equiv\left(z f^{\prime}\right) \sum_{m} m B_{n m} f^{m-1}
$$

We denote $H_{n}(w)=\sum_{m} B_{n m} w^{m}$ and then $H_{n}^{\prime}(w)=\sum_{m} m B_{n m} w^{m-1}$. Then $(1+n) H_{n}(f)=\left(z f^{\prime}\right) H_{n}^{\prime}(f)$ and hence,

$$
\left(\frac{1}{1+n}\right) \frac{H_{n}^{\prime}(f)}{H_{n}(f)}=\frac{1}{z f^{\prime}(z)} \text { independent of } n .
$$


So $\forall k, n \in \mathbb{Z}^{+} \cup\{0\},(1 /(1+n))\left(\log H_{n}(f)\right)^{\prime}=(1 /(1+k))\left(\log H_{k}(f)\right)^{\prime}$ and we deduce that $H_{n}^{1+k}=H_{k}^{1+n}$. Taking $k=0$ we get $H_{n}=H_{0}^{1+n}$. Substituting that into the identity (3.1) we obtain $(1+n) H_{0}(f)^{1+n}=$ $\left(z f^{\prime}\right)(1+n) H_{0}(f)^{1+n}$. So $H_{0}(f)=z f^{\prime}$. Thus $H_{n}=H_{0}^{1+n}=\left(z f^{\prime}\right)^{1+n}$. We recall that according to our notations we have $G(u, v)=\sum B_{n m} u^{n} v^{m}$, and hence

$$
\begin{gathered}
G(t, f)=\left.\sum_{n, m} B_{n m} u^{n} v^{m}\right|_{(u, v)=(t, f)}=\sum_{n, m} B_{n m} t^{n} f^{m}=\sum_{n}\left(\sum_{m} B_{n m} f^{m}\right) t^{n}= \\
=\sum_{n} H_{n}(f) t^{n}=\sum_{n}\left(z f^{\prime}\right)^{1+n} t^{n}=\left(z f^{\prime}\right) \sum_{n}\left(t z f^{\prime}(z)\right)^{n}=\frac{z f^{\prime}}{1-t z f^{\prime}} .
\end{gathered}
$$

We strongly use here the permanence principle. We deduce that

$$
\frac{(t z) f^{\prime}(z)}{1-(t z) f^{\prime}(z)}=g(f(t z))
$$

In the last step we used $G(t, f)=(1 / t) g(f(t z))$. In particular for $t=1$ we obtain the following functional equation,

$$
\frac{z f^{\prime}(z)}{1-z f^{\prime}(z)}=g(f(z)) \text {. }
$$

Since the right hand side is an entire function we deduce that $\forall z \in \mathbb{C}$, $z f^{\prime}(z) \neq 1$. We now recall a result from Nevanlinna's value distribution theory for meromorphic functions.

Theorem (R. Nevanlinna). Let $q(z)$ be a transcendental meromorphic function. Then for each value a, finite or infinite, the equation $q(z)=$ a has an infinite number of roots except for at most two exceptional values.

Using that with $q(z)=z f^{\prime}(z)$ we see that for each of $a=0, \infty$ the equation has exactly one root. For $a=1$ it has no roots and we deduce that $z f^{\prime}(z)$ is not a transcendental meromorphic function. This contradiction shows that our original equation

$$
\frac{1}{t} g(f(t z))=G(t, f(z)), 0<t \leq 1,
$$

is contradictory and so we can find a sequence of real numbers $t_{n} \rightarrow 1^{-}$such that $\forall n \in \mathbb{Z}^{+}$,

$$
h_{t_{n}}(z)=\frac{1}{t_{n}} g\left(f\left(t_{n} z\right)\right) \notin R_{f}(\operatorname{elh}(\mathbb{C})) .
$$


We have $\lim _{n \rightarrow \infty} h_{t_{n}}(z)=h(z)=g(f(z)) \in R_{f}(\operatorname{elh}(\mathbb{C}))$. This proves that $R_{f}(\operatorname{elh}(\mathbb{C})) \subseteq \partial R_{f}(\operatorname{elh}(\mathbb{C}))$ and so we have proved the following interesting,

Theorem 3.18. $\forall f \in \operatorname{elh}(\mathbb{C})-\operatorname{Aut}(\mathbb{C}), \partial R_{f}(\operatorname{elh}(\mathbb{C}))=R_{f}(\operatorname{elh}(\mathbb{C}))$.

It is natural to ask if for all $f \in \operatorname{elh}(\mathbb{C})$, the image $L_{f}(\operatorname{elh}(\mathbb{C}))$ is a closed subset of $\left(\operatorname{elh}(\mathbb{C}), \tau_{C C}\right)$. The key for proving the parallel claim for $R_{f}(\operatorname{elh}(\mathbb{C}))$ (Proposition 3.16 part 2) was the tame behavior of $\tau_{C C}$-convergent sequences. Namely, if $f, g_{n} \in \operatorname{elh}(\mathbb{C})$ and $\left\{g_{n} \circ f\right\}$ is $\tau_{C C}$-convergent then $\left\{g_{n}\right\}$ is $\tau_{C C}$-convergent. This tameness is false for $L_{f}$. For example, $f(z)=e^{z}$, $g_{n}(z)=z+2 \pi i n$ satisfy by the periodicity of $f, f \circ g_{n}=f$ but $\left\{g_{n}\right\}$ is not $\tau_{C C}$-convergent. Proposition 3.14 tells us that $L_{f}$ might not be injective. With the aid of Proposition 3.13 we can make this much more precise.

Proposition 3.19. $\forall f, g \in \operatorname{elh}(\mathbb{C})$ we have $L_{f}^{-1}\left(L_{f}(g)\right)=\left\{g(z)+k_{j} e^{t(z)} \mid j=\right.$ $\left.0, \ldots, N, k_{0}=0\right\}$, where $N \in \mathbb{Z}^{+} \cup\{0, \infty\}$. Moreover, we have,

$N$ is finite $\Leftrightarrow \begin{cases}L_{f}^{-1}\left(L_{f}(g)\right)=\{g\} & \left(N=0, k_{0}=0\right), \\ \text { or } & \\ \left|L_{f}^{-1}\left(L_{f}(g)\right)\right|>1 \text { in which case }: & f \in \mathbb{C}[z], N=\operatorname{deg} f-1 .\end{cases}$

\section{Proof.}

Clearly $g \in L_{f}^{-1}\left(L_{f}(g)\right)$ and by Proposition 3.13 if $h \neq g, h \in L_{f}^{-1}\left(L_{f}(g)\right)$ then $h(z)=g(z)+e^{t(z)}$ for some entire $t(z)$. Let us assume further that $h_{1}(z) \in L_{f}^{-1}\left(L_{f}(g)\right)-\{g, h\}$. Then by Proposition 3.13 there are two entire functions $t_{1}(z), t_{2}(z)$ such that $h_{1}(z)=g(z)+e^{t_{1}(z)}, h_{1}(z)=h(z)+e^{t_{2}(z)}$. Hence $g(z)+e^{t_{1}(z)}=\left(g(z)+e^{t(z)}\right)+e^{t_{2}(z)}$, and so we obtain $1 \equiv e^{t(z)-t_{1}(z)}+$ $e^{t_{2}(z)-t_{1}(z)}$. This implies that the entire functions $e^{t(z)-t_{1}(z)}$ and $e^{t_{2}(z)-t_{1}(z)}$ do not assume the values $\{0,1\}$ and hence are constant by Picard's Theorem. Thus $t_{1}(z)=t(z)+c_{1}, t_{2}(z)=t(z)+c_{2}$ for some $c_{1}, c_{2} \in \mathbb{C}$ which proves that any function in $L_{f}^{-1}\left(L_{f}(g)\right)$ is of the form $g(z)+k e^{t(z)}, k \in \mathbb{C}$. Since $\mathbb{C}$ is tiled up by a countable set of pairwise disjoint maximal domains of $f$ the set of admissible constants $k$ is a countable set, say, $\left\{k_{j} \mid j=0, \ldots, N, k_{0}=0\right\}$. Note that if $N \geq 1$ we may assume that $k_{1}=1$. The only entire functions with finitely many pairwise disjoint maximal domains are polynomials, [13, $f \in \mathbb{C}[z]$ and the number of tiles for such an $f(z)$ is $\operatorname{deg} f$. Thus in this case $N=\operatorname{deg} f-1$.

Proposition 3.20. The set $\left\{k_{j} e^{t(z)} \mid j \in I, k_{0}=0\right\}$ is a cyclic subgroup of $(\mathbb{C},+)$. 


\section{Proof.}

Given $f, g \in \operatorname{elh}(\mathbb{C})$ we saw in Proposition 3.19 that

$$
L_{f}^{-1}\left(L_{f}(g)\right)=\left\{g(z)+k_{j} e^{t(z)} \mid j \in I, k_{0}=0\right\},|I| \leq \aleph_{0} .
$$

Thus for a generic $z$, the points $g(z)+k_{j} e^{t(z)}$ are the full set of points that are $f$-equivalent to $g(z)$. However, for each $g(z)$ the set of all $f$-equivalent points to $g(z)$ are the orbit of $g(z)$ under the action of the group of the $f$-deck transformations. It is clear that the composition of the two $f$-deck transformations $g(z) \rightarrow g(z)+k_{1} e^{t(z)}, g(z) \rightarrow g(z)+k_{2} e^{t(z)}$ is $g(z) \rightarrow$ $g(z)+\left(k_{1}+k_{2}\right) e^{t(z)}$.

Proposition 3.21. If $f \in \mathbb{C}[z]$, then $L_{f}$ is injective.

\section{Proof.}

By Proposition 3.19 and Proposition 3.20 in this case $\left\{k_{j} e^{t(z)} \mid j \in I, k_{0}=0\right\}$ is a finite cyclic subgroup of $(\mathbb{C},+)$ and hence trivial.

Remark 3.22. The last statement in the proof of Proposition 3.20 follows by: Let $\left.h_{(} z\right), h_{2}(z) \in L_{f}^{-1}\left(L_{f}(g)\right)$. Then we note that we have the two identities $L_{f}^{-1}\left(L_{f}(g)\right)=L_{f}^{-1}\left(L_{f}\left(h_{1}\right)\right)=L_{f}^{-1}\left(L_{f}\left(h_{2}\right)\right)$. As explained in the proof of Proposition 3.19 there is an entire $t(z)$ and two constants $k_{1}, k_{2}$ so that $h_{1}(z)=g(z)+k_{1} e^{t(z)}, h_{2}(z)=g(z)+k_{2} e^{t(z)}$. Also by $h_{2} \in L_{f}^{-1}\left(L_{f}\left(h_{1}\right)\right)$ there exists a constant $k_{3}$ so that $h_{2}(z)=h_{1}(z)+k_{3} e^{t(z)}$ (same entire function $t(z)$ ). We can use the symbols $k_{1}, k_{2}$ and $k_{3}$ to denote the corresponding deck-transformations of $f^{-1} \circ f$. Indeed by $h_{2}(z)=h_{1}(z)+k_{3} e^{t(z)}$ we have $g(z)+k_{2} e^{t(z)}=\left(g(z)+k_{1} e^{t(z)}\right)+k_{3} e^{t(z)}=g(z)+\left(k_{1}+k_{2}\right) e^{t(z)}$, so the composition $k_{2}=k_{3} \circ k_{1}$ of the deck-transformations corresponds to complex addition $k_{2}=k_{3}+k_{1}$.

Remark 3.23. If $f \in \operatorname{elh}(\mathbb{C})-\mathbb{C}[z], g \in \operatorname{elh}(\mathbb{C})$ and $\left|L_{f}^{-1}\left(L_{f}(g)\right)\right|>1$, then by Proposition 3.19 we have $\left|L_{f}^{-1}\left(L_{f}(g)\right)\right|=\aleph_{0}$ so by Proposition 3.20 the set $\left\{k_{j} e^{t(z)} \mid j \in I, k_{0}=0\right\}$ is an infinite cyclic group for addition. But then by changing, if necessary, the entire $t(z)$ we may assume that the set is, in fact $\left\{j e^{t(z)} \mid j \in \mathbb{Z}\right\}$.

Proposition 3.24. $\forall f \in \operatorname{elh}(\mathbb{C})-\mathbb{C}[z], g \in \operatorname{elh}(\mathbb{C})$ if $\left|L_{f}^{-1}\left(L_{f}(g)\right)\right|>1$ then there exists an entire $t(z)$ in the variable $z$ and an entire $h(z, w)$ in $(z, w)$ so that

$$
f\left(g(z)+w e^{t(z)}\right)=f(g(z))+e^{h(z, w)} \sin \pi w .
$$

\section{Proof.}

We consider $f\left(g(z)+w e^{t(z)}\right)-f(g(z))$ with the entire function $t(z)$ of 
Proposition 3.19 normalized as in the last remark. Then this function is entire in $(z, w)$ and for a fixed $z$ it vanishes exactly for $w=j \in \mathbb{Z}$. Since $\sum_{j=1}^{\infty}(1 / j)=\infty, \sum_{j=1}^{\infty}\left(1 / j^{2}\right)<\infty$ we get by writing the Weierstrass canonical infinite product for our function,

$$
\begin{gathered}
f\left(g(z)+w e^{t(z)}\right)-f(g(z))=e^{h_{0}(z, w)} w \prod_{j \neq 0, j=-\infty}^{\infty}\left(1-\frac{w}{j}\right) e^{w / j}= \\
=e^{h_{0}(z, w)} w \prod_{j=1}^{\infty}\left(1-\frac{w^{2}}{j^{2}}\right)=\frac{1}{\pi} e^{h_{0}(z, w)} \sin \pi w
\end{gathered}
$$

where in the last step we used the infinite product representation of $\sin \pi w$ which extends the Euler-Wallis' formula for $\pi$ :

$$
\frac{\pi}{2}=\frac{2}{1} \cdot \frac{2}{3} \cdot \frac{4}{3} \cdot \frac{4}{5} \cdot \frac{6}{5} \cdot \frac{6}{7} \cdot \ldots=\prod_{j=1}^{\infty}\left(\frac{4 j^{2}}{4 j^{2}-1}\right) .
$$

Example 3.25. $f(z)=e^{2 \pi i z}, g(z)=e^{z}, L_{f}^{-1}\left(L_{f}(g)\right)=\left\{e^{z}+j \mid j \in \mathbb{Z}\right\}=$ $\left\{e^{z}+j e^{0} \mid j \in \mathbb{Z}\right\}$. So in this case the normalized entire $t(z) \equiv 0$, and $f\left(g(z)+w e^{t(z)}\right)=f\left(e^{z}+w\right)=e^{2 \pi i\left(e^{z}+w\right)}=e^{2 \pi i e^{z}} e^{2 \pi i w}=e^{2 \pi i e^{z}}(1+$ $\left.\left(e^{2 \pi i w}-1\right)\right)=f(g(z))+e^{2 \pi i e^{z}}\left(e^{2 \pi i w}-1\right)=f(g(z))+e^{2 \pi i e^{z}}[(\cos 2 \pi w-$ 1) $+i(\sin 2 \pi w]=f(g(z))+e^{2 \pi i e^{z}}\left[-2 \sin ^{2} \pi w+i 2 \cos \pi w \sin \pi w\right]=f(g(z))+$ $2 i e^{2 \pi i e^{z}}(\cos \pi w+i \sin \pi w) \sin \pi w=f(g(z))+2 i e^{\pi i\left(2 e^{z}+w\right)} \sin \pi w=f(g(z))+$ $2 i e^{\pi i\left(2 e^{z}+w\right)} \sin \pi w=f(g(z))+e^{\log 2+\pi i\left((1 / 2)+2 e^{z}+w\right)} \sin \pi w$. Thus in this case $t(z) \equiv 0$ and $h(z, w)=\log 2+\pi i\left((1 / 2)+2 e^{z}+w\right)$.

Proposition 3.26. Let $f \in \operatorname{elh}(\mathbb{C})-\mathbb{C}[z], g \in \operatorname{elh}(\mathbb{C})$ satisfy $\left|L_{f}^{-1}\left(L_{f}(g)\right)\right|>$ 1 and let $t(z), h(z, w)$ be the entire functions for which

$$
f\left(g(z)+w e^{t(z)}\right)=f(g(z))+e^{h(z, w)} \sin \pi w \quad \text { (Proposition) 3.24. }
$$

Then there exists an entire $k(z, w)$ in $(z, w)$ so that

$$
\frac{\partial h}{\partial w}(z, w) \cdot \sin \pi w+\pi \cos \pi w=e^{k(z, w)} .
$$

Proof.

We differentiate with respect to $w$ the functional equation $f\left(g(z)+w e^{t(z)}\right)=$ $f(g(z))+e^{h(z, w)} \sin \pi w$. We obtain

$$
e^{t(z)} f^{\prime}\left(g(z)+w e^{t(z)}\right)=\frac{\partial h}{\partial w}(z, w) e^{h(z, w)} \sin \pi w+\pi e^{h(z, w)} \cos \pi w,
$$




$$
\frac{\partial h}{\partial w}(z, w) \sin \pi w+\pi \cos \pi w=e^{t(z)-h(z, w)} f^{\prime}\left(g(z)+w e^{t(z)}\right) .
$$

Since $f \in \operatorname{elh}(\mathbb{C})$ it follows that $f^{\prime}$ never vanishes. Hence the entire function

$$
\frac{\partial h}{\partial w}(z, w) \cdot \sin \pi w+\pi \cos \pi w,
$$

never vanishes and we are done.

Remark 3.27. Proposition 3.21 has a truly trivial proof. For by the Fundamental Theorem of the Algebra $f \in \mathbb{C}[z] \cap \operatorname{elh}(\mathbb{C}) \Leftrightarrow f \in \operatorname{Aut}(\mathbb{C})$.

we can sharpen the statement of Proposition 3.24 as follows,

Proposition 3.28. $\forall f \in \operatorname{elh}(\mathbb{C})-\mathbb{C}[z], g \in \operatorname{elh}(\mathbb{C})$ if $\left|L_{f}^{-1}\left(L_{f}(g)\right)\right|>1$ then there exist three entire functions $t(z), h(z)$ and $L(w)$ so that

$$
f\left(g(z)+w e^{t(z)}\right)=f(g(z))+e^{L(w)+h(z)} \sin \pi w .
$$

\section{Proof.}

we need to prove that the entire function $h(z, w)$ has the structure $h(z, w)=$ $L(w)+h(z)$ (we abuse the notation $h$ ). By Proposition 3.26 we have

$$
\frac{\partial h}{\partial w}(z, w) \cdot \sin \pi w+\pi \cos \pi w=e^{k(z, w)} .
$$

Let $L(z, w)=(1 / \pi)(\partial h / \partial w)$. Then we conclude that $L(z, w) \sin \pi w+\cos \pi w$ never vanishes. By changing our notation for $k(z, w)$ we conclude that $L(z, w) \sin \pi w+\cos \pi w=e^{k(z, w)} \Rightarrow L_{z} \sin \pi w=k_{z} e^{k}$. Let us assume in order to get a contradiction that $L_{z} \not \equiv 0$. Then also $k_{z} \not \equiv 0$ and so $\left(L_{z} / k_{z}\right) \sin \pi w=e^{k}$. Thus

$$
\begin{gathered}
\frac{L_{z}}{k_{z}} \sin \pi w=L \sin \pi w+\cos \pi w \\
\Rightarrow\left\{\begin{aligned}
\left(L-L_{z} / k_{z}\right) \sin \pi w+\cos \pi w & \equiv \\
\sin \pi w+\cos \pi w & =e^{\theta(w)}
\end{aligned}\right.
\end{gathered}
$$

The second equation is a consequence of $\sin ^{2} \pi w+\cos ^{2} \pi w \equiv 1$. We can consider the last system as a linear system in the unknowns $\sin \pi w$ and $\cos \pi w$. This system is non-homogeneous, the equations are independent and it is consistent $\forall(z, w) \in \mathbb{C}^{2}$. Hence the coefficients matrix never vanishes. Thus

$$
\left|\begin{array}{cc}
L-L_{z} / k_{z} & 1 \\
1 & 1
\end{array}\right|=e^{k_{1}(z, w)}
$$




$$
\Rightarrow\left(L-\frac{L_{z}}{k_{z}}\right)-1=e^{k_{1}} .
$$

Multiplying the last equation by $\sin \pi w$ gives

$$
\begin{gathered}
\left(L-\frac{L_{z}}{k_{z}}\right) \sin \pi w-\sin \pi w=e^{k_{1}} \sin \pi w, \\
\Rightarrow L \sin \pi w-\sin \pi w-\frac{L_{z}}{k_{z}} \sin \pi w=e^{k_{1}} \sin \pi w, \\
\Rightarrow L \sin \pi w-\sin \pi w-(L \sin \pi w+\cos \pi w)=e^{k_{1}} \sin \pi w, \\
\Rightarrow-\sin \pi w-\cos \pi w=e^{k_{1}} \sin \pi w .
\end{gathered}
$$

This is a contradiction as the substitution $w=0$ shows. This shows that our assumption $L_{z} \not \equiv 0$ is contradictory. Hence $L_{z}(z, w) \equiv 0$ and so $L(z, w)=$ $L(w)$ depends on $w$ only. Thus

$$
\frac{\partial h}{\partial w}(z, w)=\pi L(w)
$$

So $h(z, w)=L(w)+h(z)$.

Remark 3.29. The equation in the last proposition can be written as follows,

$$
e^{-h(z)} f\left(g(z)+w e^{t(z)}\right)=e^{-h(z)} f(g(z))+e^{L(w)} \sin \pi w .
$$

If we differentiate this with respect to $w$ we get,

$$
e^{t(z)-h(z)} f^{\prime}\left(g(z)+w e^{t(z)}\right)=\left(L^{\prime}(w) \sin \pi w+\pi \cos \pi w\right) e^{L(w)} .
$$

Since $f^{\prime}$ never vanishes (because $f \in \operatorname{elh}(\mathbb{C})$ ) we deduce (in accordance to the proof of Proposition 3.28) that

$$
L^{\prime}(w) \sin \pi w+\pi \cos \pi w=e^{k(w)} .
$$

In fact $e^{k(w)}=c \cdot e^{-L(w)}$ because the two sides of the equation above must be constant (the left hand side depends only on $z$ while the right hand side depends only on $w$ ). If we differentiate the identity in Remark 3.29 with respect to $z$ we get,

$$
-h^{\prime} f\left(g+w e^{t}\right)+\left(g^{\prime}+w t^{\prime} e^{t}\right) f^{\prime}\left(g+w e^{t}\right)=-h^{\prime} f(g)+g^{\prime} f^{\prime}(g) .
$$

This proves the following, 
Proposition 3.30. Under the condition of Proposition 3.28 we have:

a) $L^{\prime}(w) \sin \pi w+\pi \cos \pi w$ never vanishes.

b) The function $-h^{\prime}(z) f\left(g(z)+w e^{t(z)}\right)+\left(g^{\prime}(z)+w t^{\prime}(z) e^{t(z)}\right) f^{\prime}\left(g(z)+w e^{t(z)}\right)$ is independent of $w$.

If in part (b) we substitute $w=-g(z) e^{-t(z)}$ and recall that $f(0)=0$, $f^{\prime}(0)=1$ we get after the simplification $g^{\prime}(z)-g(z) t^{\prime}(z)$. We conclude the following,

Proposition 3.31. $-h^{\prime}(z) f(g(z))+g^{\prime}(z) f^{\prime}(g(z)) \equiv g^{\prime}(z)-g(z) t^{\prime}(z)$.

We can do better by differentiating the function in Proposition 3.30 part (b)with respect to $w$,

Proposition 3.32. $\left(t^{\prime}(z)-h^{\prime}(z)\right) f^{\prime}\left(g(z)+w e^{t(z)}\right)+\left(g^{\prime}(z)+w t^{\prime}(z) e^{t(z)}\right) f^{\prime \prime}(g(z)+$ $\left.w e^{t(z)}\right) \equiv 0$.

Theorem 3.33. Let $f \in \operatorname{elh}(\mathbb{C})$. Then $L_{f}$ is not injective if and only if

$$
f(z)=\frac{1}{b} e^{b z}+a \text { for some } a \in \mathbb{C}, b \in \mathbb{C}^{\times} .
$$

\section{Proof.}

Clearly $L_{e^{b z / b+a}}$ is not injective. In the other direction, if $L_{f}$ is not injective we have the identity of Proposition 3.32. First, we claim that $t^{\prime}(z) \equiv 0$. If not, then we can substitute $w=-g^{\prime}(z) e^{-t(z)} / t^{\prime}(z)$ into that identity. Since $f^{\prime}$ never vanishes we conclude that $t^{\prime}(z)-h^{\prime}(z) \equiv 0$. Hence $\left(g^{\prime}(z)+\right.$ $\left.\left.w t^{\prime}(z) e^{t(z)}\right) f^{\prime \prime} * g(z)+w e^{t(z)}\right) \equiv 0$. Now $f^{\prime \prime}\left(g(z)+w e^{t(z)}\right) \not \equiv 0$ because $w$ and $z$ are independent and $e^{t(z)} \neq 0$ and so in that case we had to conclude that $f^{\prime \prime}(z) \equiv 0$. But then $f \in \mathbb{C}[z] \cap \operatorname{elh}(\mathbb{C})$ so by Proposition $3.21 L_{f}$ is injective. Thus $g^{\prime}(z)+w t^{\prime}(z) e^{t(z)} \equiv 0$ which in turn (if $w=0$ ) implies that $g^{\prime}(z) \equiv t^{\prime}(z) \equiv 0$. This contradicts our assumption that $t^{\prime}(z) \not \equiv 0$. This proves that $t^{\prime}(z) \equiv 0$ so that $t(z) \equiv c$ a constant. We get from the identity in Proposition 3.32,

$$
-h^{\prime}(z) f^{\prime}\left(g(z)+w e^{c}\right)+g^{\prime}(z) f^{\prime \prime}\left(g(z) w e^{c}\right) \equiv 0 .
$$

We now substitute $w=-g(z) e^{-c}$ and obtain $h^{\prime}(z) f^{\prime}(0)+g^{\prime}(z) f^{\prime \prime}(0) \equiv 0$. We recall that $f^{\prime}(0)=1$ and get $g(z)=\alpha h(z)+\beta, \alpha, \beta \in \mathbb{C}$. So

$$
-h^{\prime}(z) f^{\prime}\left(g(z)+w e^{c}\right) \alpha h^{\prime}(z) f^{\prime \prime}\left(g(z)+w e^{c}\right) \equiv 0 .
$$

Now $g(z) \in \operatorname{elh}(\mathbb{C})$ and so $g^{\prime}(z) \not \equiv 0$ and $h^{\prime}(z) \not \equiv 0$. Thus,

$$
-f^{\prime}\left(g(z)+w e^{c}\right)+\alpha f^{\prime \prime}\left(g(z)+w e^{c}\right) \equiv 0 .
$$

We conclude that $f^{\prime}(z)=\alpha f^{\prime \prime}(z)$ for some $\alpha \in \mathbb{C}^{\times}$. Hence $f(z)=\alpha f^{\prime}(z)+\delta$ and so $f(z)=e^{b z} / b+a$. 
Remark 3.34. Theorem 3.33 tells us that the only obstacle for $L_{f}$ to be injective for any $f \in \operatorname{elh}(\mathbb{C})$ is that $f$ is a translation of a conjugation by a $b \in \mathbb{C}^{\times}$of the exponential function, $e^{z}$.

Remark 3.35. Let us consider again Proposition 3.30 part (b), with $t(z) \equiv c$ a constant. Then the following is independent of $w$ :

$$
\left.\left.-h^{\prime}\right) z\right) f\left(g(z)+w e^{c}\right)+g^{\prime}(z) f^{\prime}\left(g(z)+w e^{c}\right) .
$$

At this point we know that $f(z)=e^{z}+a$ (we took $b=1$ for simplicity). So

$$
-h^{\prime}(z)\left(e^{g(z)+w e^{c}}+a\right)+g^{\prime}(z) f^{\prime}\left(g(z)+w e^{c}\right),
$$

is independent of $w$. The above equals

$$
e^{w e^{c}}\left(-h^{\prime}(z) e^{g(z)}+g^{\prime}(z) e^{g(z)}\right)-h^{\prime}(z) a,
$$

and we conclude that $-h^{\prime}(z)+g^{\prime}(z) \equiv 0$ which is consistent with the proof of Theorem 3.33.

Remark 3.36. For later needs we point to the fact that, unfortunately, the family elh $(\mathbb{C})$ is not a normal family. For example we may consider the orbit of $e^{z}$ under conjugation with $\mathbb{Z}^{+}$, i.e. the sequence

$$
\left\{\frac{1}{n} e^{n z}\right\}_{n=1}^{\infty} .
$$

This sequence is contained in $\operatorname{elh}(\mathbb{C})$ but has no convergent subsequence (uniformly on compacta). For if $z=x \in \mathbb{R}^{+}$then $\lim _{n \rightarrow \infty} e^{n z} / n=\infty$ while if $z=x \in \mathbb{R}^{-} \cup\{0\}$ then $\lim _{n \rightarrow \infty} e^{n z} / n=0$ and for $y \in \mathbb{R}$ which is rationally independent of $\pi$ the numbers $e^{\log n+i y} / n$ form a dense subset of $|z|=1$ (in the standard topology).

\section{References}

[1] H. Bass, E. Connell and D. Wright, The Jacobian Conjecture: Reduction of Degree and Formal Expansion of the Inverse, Bulletin of the American Mathematical Society, 7(1982), 287-330.

[2] L. M. Druzkowsky, An effective Approach to Keller's Jacobian Conjecture, Math. Ann., 264(1983), 303-313.

[3] L. M. Druzkowsky, The Jacobian Conjecture in case of rank or corank less than three, J. of Pure and Applied Algebra, 85(1993), 233-244. 
[4] A. van den Essen (ed.), Automorphisms of affine Spaces, Curacao, Caribbean Mathematics Foundation, Kluwer Academic Publishers, July 4-8, 1994, 1995, Proceedings of the conference 'Invertible Polynomial Maps'.

[5] A. van den Essen, Polynomial Automorphisms and the Jacobian Conjecture, Progress in Mathematics, Volume 190, Birkhäuser Verlag, Basel-Boston-Berlin, 2000.

[6] J. Hadamard, Sur les transformations pontuelles, Bull. de la Soc. Math. de France, t 34, 1906.

[7] T. Kambayashi, Pro-affine Algebras, Ind-affine Groups and the Jacobian Problem, Journal of Algebra 185, 481-501, 1996.

[8] T. Kambayashi, Some basic results on pro-affine algebras and ind-affine schemes, Osaka J. Math. 40, 621-638, 2003.

[9] T. Kambayashi, Inverse limits of polynomial rings, Osaka J. Math. 41, 617-624, 2004.

[10] O. H. Keller, Ganze Cremona-Transformationen, Monatsh. Math. Phys., 47(1939), 299-306.

[11] Shrawan Kumar, Kac-Moody Groups, their Flag Varieties and Representation Theory,Progress in Math., Vol. 204, Birkhäuser Verlag, Boston-Basel-Berlin, 2002.

[12] T.-T. Moh, On the global Jacobian Conjecture and the Configuration of root, J. Reine Angew. Math., 340(1983), 140-212.

[13] R. Peretz, Maximal domains for entire functions, J. d'Analyse Mathematique, 1990.

[14] R. Peretz, The geometry of the asymptotics of polynomial maps, The Israel Journal of Mathematics, Vol. 105(1998), 1-59.

[15] Ronen Peretz, The Jacobian variety, Acta Mathematica Vietnamica, Vol. 32, No. 3, 95-105, 2007.

[16] Ronen Peretz, The 2-D Jacobian Conjecture, the $d$-inversion approximation and its natural boundary, Zapiski POMI (St. Petersburg Department of V.A. Steklov Institute of Mathematics of the Russian Academy of Sciences), Tom 373, 210-225, 2009. English translation is published in The Journal of Mathematical Sciences, Springer. 
[17] S. Pinchuk, A counterexample to the real Jacobian Conjecture, Math. Zeitschrift, 217(1994), 1-4.

[18] J. Randall, The real Jacobian problem, Proceedings of Symposia in Pure Mathematics, 40(1983),411-414.

[19] I. R. Shafarevich, On some infinite-dimensional groups, Rend. Mat. Appl. 25, 208-212, 1966.

[20] I. R. Shafarevich, On some infinite-dimensional groups-II, Math. USSR - Izvestija 18, 185-194, 1982 (Cf. Math. Reviews 84a, 1984, 14021.).

[21] S. Smale, Mathematical problems for the next century, Math. Intellingecer, 20(1998), No. 2, 7-15.

[22] A. V. Yagzhev, On Keller's problem, Siberian Math. J., 21(1980), 747754 .

Ronen Peretz

Department of Mathematics

Ben Gurion University of the Negev

Beer-Sheva , 84105

Israel

E-mail: ronenp@math.bgu.ac.il 